\title{
Induction of antigen-specific cytotoxic $T$ lymphocytes by fusion cells generated from allogeneic plasmacytoid dendritic and tumor cells
}

\author{
SHIGEO KOIDO ${ }^{1-3}$, SADAMU HOMMA ${ }^{3}$, SHIN KAN $^{3}$, KAZUKI TAKAKURA ${ }^{1}$, YOSHIHISA NAMIKI ${ }^{2}$, \\ HIROKO KOBAYASHI $^{1}$, ZENSHO ITO $^{1}$, KAN UCHIYAMA ${ }^{1}$, MIKIO KAJIHARA ${ }^{1}$, SEIJI ARIHIRO ${ }^{1}$, \\ HIROSHI ARAKAWA ${ }^{1}$, MASATO OKAMOTO ${ }^{4}$, TOSHIFUMI OHKUSA ${ }^{1}$, \\ JIANLIN GONG $^{5}$ and HISAO TAJIRI ${ }^{1}$
}

\begin{abstract}
${ }^{1}$ Division of Gastroenterology and Hepatology, Department of Internal Medicine, ${ }^{2}$ Institute of Clinical Medicine and Research, Jikei University School of Medicine, Chiba 277-8564; ${ }^{3}$ Department of Oncology, Jikei University School of Medicine, Tokyo 105-8461; ${ }^{4}$ Division of Cellular Signaling, Institute for Advanced Medical Research, Keio University School of Medicine, Tokyo 160-8582, Japan; ${ }^{5}$ Department of Medicine, Boston University School of Medicine, Boston, MA 02118, USA
\end{abstract}

Received February 20, 2014; Accepted April 17, 2014

DOI: 10.3892/ijo.2014.2433

\begin{abstract}
Previous work has demonstrated that fusion cells generated from autologous monocyte-derived dendritic cells (MoDCs) and whole tumor cells induce efficient antigenspecific cytotoxic $\mathrm{T}$ lymphocytes. A major limitation to the use of this strategy is the availability of adequate amounts of autologous tumor cells. Moreover, MoDCs from cancer patients are often defective in their antigen-processing and presentation machinery. In this study, two types of allogeneic cells, a leukemia plasmacytoid dendritic cell (pDC) line (PMDC05) and pancreatic cancer cell lines (PANC-1 or MIA $\mathrm{PaCa}-2)$, were fused instead of autologous MoDCs and tumor cells. We created four types of pDC/tumor fusion cells by alternating fusion partners and treating with lipopolysaccharide (LPS): i) PMDC05 fused with PANC-1 (pDC/PANC-1), ii) PMDC05 fused with MIA PaCa-2 (pDC/MIA PaCa-2), iii) LPS-stimulated pDC/PANC-1 (LPS-pDC/PANC-1) and iv) LPS-stimulated pDC/MIA PaCa-2 (LPS-pDC/MIA $\mathrm{PaCa}-2)$ and examined their antitumor immune responses. The LPS-pDC/tumor cell fusions were the most active, as demonstrated by their: i) upregulated expression of HLA-DR and CD86 on a per-fusion-cell basis, ii) increased production of IL-12p70, iii) generation of a higher percentage of IFN- $\gamma$ producing $\mathrm{CD}^{+}$and $\mathrm{CD}^{+}$T cells and iv) augmented induction of MUC1-specific $\mathrm{CD}^{+} \mathrm{T}$ cells that lyse target tumor cells.
\end{abstract}

Correspondence to: Dr Shigeo Koido, Department of Oncology, Jikei University School of Medicine, 163-1 Kashiwa-shita, Kashiwa, Chiba 277-8564, Japan

E-mail: shigeo_koido@jikei.ac.jp

Key words: fusion, plasmacytoid dendritic cell, cytotoxic $\mathrm{T}$ lymphocyte, MUC1
This study provides the first evidence for an in vitro induction of antigen-specific cytotoxic T lymphocytes by LPS-stimulated fusion cells generated from leukemia plasmacytoid DCs and tumor cells and suggests that this strategy has potential applicability to the field of adoptive immunotherapy.

\section{Introduction}

Dendritic cells (DCs) are specialized antigen-presenting cells (APCs) that play a critical role in the induction of primary immune responses (1). Therefore, several strategies have been developed to deliver tumor-associated antigens (TAAs) to autologous monocyte-derived dendritic cells (MoDCs) for the induction of efficient antigen-specific cytotoxic $\mathrm{T}$ lymphocytes (CTLs). One of the strategies is the administration of fusion cells generated from MoDCs and whole tumor cells (2). In MoDC/tumor fusions, a broad array of TAAs, including known and unidentified molecules, are delivered to MoDCs, processed, and presented to $\mathrm{CD}^{+}$and $\mathrm{CD}^{+} \mathrm{T}$ cells in complex with $\mathrm{MHC}$ class I and II molecules and in the context of co-stimulatory signals $(3,4)$. Moreover, MoDCs and tumor cells can be independently subjected to manipulations for the acquisition of desired characteristics that persist after fusion (4).

A major limitation to the use of MoDC/tumor fusions is the availability of adequate amounts of autologous tumor cells, which stems from the limited availability of viable tumor samples and/or technical difficulties in cancer cell culture. Moreover, MoDCs from advanced cancer patients may be defective in their antigen-processing and presentation machinery due to the presence of tumor-derived immune suppressive molecules or as a result of chemotherapy (5). To circumvent all of these issues, allogeneic DC and tumor cell lines can be used instead of autologous cells. Cell lines that are well characterized can be massively propagated in vitro under good manufacturing practice (GMP) standards. Thus, 
unlimited amounts of DC/tumor fusion cells can be readily available.

As APCs, plasmacytoid DCs (pDCs) have not been used extensively in cancer vaccines thus far because they are more difficult to isolate from human blood monocytes and to obtain in sufficient quantities; however, they are more efficient than MoDCs in triggering antitumor immune responses (6-8). Moreover, pDCs differ from MoDCs in many aspects, such as TLR expression, and are capable of antigen capture, processing and presentation (9,10). A human leukemia pDC line (PMDC05) was recently generated $(11,12)$ and tested for its capacity to induce effective antigen-specific CTLs upon peptide pulsing $(13,14)$. However, little is known about whether antigen-specific CTLs can be induced by pDC/tumor fusion cells.

Here, we show that fusions generated with a pDC line and a pancreatic cancer cell line expressing MUC1 antigens induce MUC1-specific CTLs in vitro. Moreover, significantly augmented MUC1-specific CTLs are induced by lipopolysaccharide (LPS)-stimulated pDC/tumor fusion cells in vitro compared with unstimulated pDC/tumor fusion cells. By selecting cancer cell lines that express the same TAAs as autologous tumor cells, pDC/tumor fusions can be made from cells that are available in the laboratory, without the use of any patient or donor materials and avoiding the constraints of autologous cells.

\section{Materials and methods}

Cells and conditioned medium. PANC-1 (MUC1 ${ }^{+}, \mathrm{HLA}_{-} 2^{+}$, HLA-A24-), MIA PaCa-2 (MUC1 ${ }^{+}$, HLA-A2-, HLA-A24 ${ }^{+}$) and K562 $\left(\mathrm{MUC1}^{+}\right.$, HLA-A2-, HLA-A24-) cells were purchased from the American Type Culture Collection (ATCC, Manassas, VA, USA) and maintained in Dulbecco's modified Eagle's medium (DMEM) supplemented with $100 \mathrm{U} / \mathrm{ml}$ penicillin, $100 \mathrm{mg} / \mathrm{ml}$ streptomycin and 10\% fetal calf serum (FCS) (15). The leukemic pDC line PMDC05 was kindly gifted from Dr Takahashi (Laboratory of Hematology and Oncology, Graduate School of Health Sciences, Niigata University, Niigata, Japan). The PMDC05 cells were cultured at a cell concentration of $1 \times 10^{6} / \mathrm{ml}$ in Iscove's modified Dulbecco's medium (IMDM) supplemented with $100 \mathrm{U} / \mathrm{ml}$ penicillin, $100 \mathrm{mg} / \mathrm{ml}$ streptomycin and $10 \% \mathrm{FCS}$.

Fusion of pDCs and tumor cells. We developed four types of $\mathrm{pDC} /$ tumor fusions by alternating fusion cell partners and treating LPS as follows: PMDC05 fused with PANC-1 (pDC/PANC-1), PMDC05 fused with MIA PaCa-2 (pDC/MIA PaCa-2), PMDC05 fused with PANC-1 in the presence of LPS (LPS-pDC/PANC-1) and PMDC05 fused with MIA PaCa-2 in the presence of LPS (LPS-pDC/MIA PaCa-2). Briefly, pancreatic cancer cells (PANC-1 or MIA PaCa-2) were mixed with pDCs (PMDC05) at a ratio of 1:1, and fusion cells were generated using $50 \%$ polyethylene glycol (PEG) (Sigma-Aldrich, St. Louis, MO) (3). The fusion cells were maintained in DMEM with or without $0.1 \mathrm{~g} / \mathrm{ml}$ LPS (Sigma-Aldrich). After 3 days of culture, the fusion cell preparations were integrated to a single entity and purified by gentle pipetting (16).

Phenotype analysis. Cells were incubated with FITC-conjugated monoclonal antibodies (mAbs) against MUC1 (CD227 clone
HMPV; BD Pharmingen, San Jose, CA), MHC class I (W6/32), MHC class II (HLA-DR), B7-1 (CD80), B7-2 (CD86), CD83 (BD Pharmingen), HLA-A2 and HLA-A24 (One Lambda, Canoga Park, CA) or matched isotype control IgG. The pDC populations were gated based on their forward- vs. side-scatter profile and then analyzed for their expression of HLA-ABC, HLA-DR, CD80, CD86, CD83 and MUC1. For analysis of dual expression in the fusion cell preparations, the cells were incubated with a FITC-conjugated $\mathrm{mAb}$ against MUC1 and PE-conjugated mAbs against HLA-DR and CD86. After the cell aggregates were gated out (16), the fused cells were identified as MUC1 + HLA-DR ${ }^{+}$or MUC1 + $\mathrm{CD}^{+} 6^{+}$using a FACScan flow cytometer (Becton-Dickinson, Mountain View, CA) and FlowJo analysis software (TreeStar, OR, USA).

$T$ cell stimulation. The study protocol was reviewed and approved by the ethics committee of the Institutional Review Board of the Jikei University School of Medicine as well as the clinical study committee of the Jikei University Kashiwa Hospital [No. 14-60 (3209)]. Peripheral blood mononuclear cells (PBMCs) from whole blood (HLA-A2 ${ }^{+}$and HLA-A24 ${ }^{+}$) were obtained with written informed consent from each individual. Briefly, PBMCs were prepared by Ficoll density gradient centrifugation and incubated in tissue culture flasks at $37^{\circ} \mathrm{C}$ for $30 \mathrm{~min}$ in Roswell Park Memorial Institute (RPMI) 1640 medium supplemented with $1 \%$ heat-inactivated autologous serum. After incubation for $60 \mathrm{~min}$ at $37^{\circ} \mathrm{C}$ to allow for adherence, the non-adherent cells were cultured with $\mathrm{pDC} /$ tumor fusion cells, $\mathrm{pDC}$ or tumor cells. The number of $\mathrm{pDC} /$ tumor fusion cells was determined based on the number of cells that coexpressed HLA-DR and MUC1 in the fusion cell preparations. Equal numbers of each type of $\mathrm{pDC} /$ tumor fusion cell (HLA-A2 ${ }^{+}$and HLA-A24 $4^{+}$) were cocultured with the non-adherent PBMCs (HLA-A2 ${ }^{+}$and HLA-A24 ${ }^{+}$) at a ratio of $1: 10$ in the absence of recombinant human (rh)IL-2 for 3 days and then purified through nylon wool to remove the APCs. A low dose of rhIL-2 (10 U/ml; Shionogi, Osaka, Japan) was added on Day 4 and maintained until Day 8. pDCs, tumor cells and pDCs mixed with tumor cells were used as controls.

Enzyme-linked immunosorbent assay (ELISA). pDC/tumor fusion cells $\left(1 \times 10^{5}\right.$ cells $/ \mathrm{ml} /$ well $)$ or $\mathrm{pDCs}\left(1 \times 10^{5}\right.$ cells $/ \mathrm{ml} /$ well $)$ were cultured for $48 \mathrm{~h}$, and their supernatants were tested for IL-12p70 and IL-10 expression (R\&D Systems, Minneapolis, $\mathrm{MN})$. The minimum detectable concentration of human IL-12p70 is typically $<0.5 \mathrm{pg} / \mathrm{ml}$.

Proliferation assay. Stimulated T cells were harvested by nylon wool separation and cultured in 96-well U-bottomed culture plates at $7 \times 10^{4}$ cells/well for 1 day. Dye solution was added to each well and incubated for $4 \mathrm{~h}$ according to the protocol of the Cell Titer 96 Non-radioactive Cell Proliferation Assay kit (Promega, Madison, WI). For measurement of proliferating $\mathrm{T}$ cells, we used a Microplate Imaging System (Bio-Rad, Hercules, CA) at an OD of $550 \mathrm{~nm}$.

IFN- $\gamma$-producing $C D 4^{+}$and $C D 8^{+} T$ cells. Stimulated T cells were harvested by nylon wool separation, and their human IFN- $\gamma$ production was analyzed using an IFN- $\gamma$ secretion 
assay kit (Miltenyi Biotec, Auburn, CA) according to the manufacturer's instructions. Briefly, the T cells were incubated with IFN- $\gamma$ catching reagent for $5 \mathrm{~min}$ at $4^{\circ} \mathrm{C}$ and then cultured for $45 \mathrm{~min}$. Next, the cells were stained with a PE-conjugated anti-IFN- $\gamma \mathrm{mAb}$ and FITC-conjugated $\mathrm{mAbs}$ against $\mathrm{CD} 4$ and CD8 (BD Pharmingen), washed, fixed with $2 \%$ paraformaldehyde and analyzed by flow cytometry using FlowJo analysis software. The $\mathrm{T}$ cell populations were gated based on their forward- vs. side-scatter profile. The $\mathrm{CD} 4^{+}$and $\mathrm{CD} 8^{+} \mathrm{T}$ cell populations were each gated, and then the percentages of IFN- $\gamma$-positive $\mathrm{CD}^{+}{ }^{+}$and $\mathrm{CD} 8^{+} \mathrm{T}$ cells among the whole $\mathrm{CD} 4^{+}$ and $\mathrm{CD}^{+} \mathrm{T}$ cell populations were calculated.

MUC1 pentamer staining. Stimulated T cells were harvested by nylon wool separation and then incubated with a PE-conjugated MUC1 pentamer (HLA-A2, STAPPVHNV) (Proimmune, Oxford, UK) for $1 \mathrm{~h}$ at $4^{\circ} \mathrm{C}$. After washing, the $\mathrm{T}$ cells were stained with a FITC-conjugated $\mathrm{mAb}$ against CD8 (BD Pharmingen), washed, fixed with $2 \%$ paraformaldehyde and analyzed by flow cytometry using FlowJo analysis software. Complexes of PE-irrelevant pentamers were used as controls. The $\mathrm{T}$ cell populations were gated based on their forward- vs. side-scatter profile. The $\mathrm{CD} 8^{+} \mathrm{T}$ cell populations were gated, and then the percentage of MUC1 pentamer-positive $\mathrm{CD}^{+} \mathrm{T}$ cells among the whole $\mathrm{CD} 8^{+} \mathrm{T}$ cell population was calculated.

Cytotoxicity assays. The cytotoxicity assays were performed by flow cytometric analysis using Active Caspase-3 Apoptosis kit I (BD Pharmingen), which measures CTL-induced caspase-3 activation in target cells by detecting the specific cleavage of fluorogenic caspase-3 (17). Briefly, the target cells were labeled with PKH-26 (Sigma-Aldrich), washed, cultured with stimulated $\mathrm{T}$ cells for $2 \mathrm{~h}$ at $37^{\circ} \mathrm{C}$ in 96 -well V-bottomed plates at the indicated effector cell:T cell (E:T) ratios. The cells were then fixed with Cytofix/Cytoperm Solution (BD Pharmingen), washed with Perm/Wash Buffer (BD Pharmingen) and incubated with a FITC-conjugated $\mathrm{mAb}$ against human active caspase-3 (BD Pharmingen) for $30 \mathrm{~min}$ at room temperature, followed by two washes with Perm/Wash buffer. In certain experiments, the tumor target cells were preincubated with anti-HLA-ABC $\mathrm{mAb}$ (W6/32; 1:100 dilution) or control $\mathrm{IgG}$ for $30 \mathrm{~min}$ at $37^{\circ} \mathrm{C}$ before adding the effector cells. The percentage of cytotoxicity (mean $\pm \mathrm{SD}$ of three replicates) was determined using the following equation: percentage of caspase- 3 staining $=($ caspase $-3+\mathrm{PKH}-26+$ cells $) /$ (caspase-3+PKH-26+cells + caspase-3-PKH-26+cells) x100.

Statistical analysis. The results are expressed as means \pm SD, as indicated in the legends. One-way analysis of variance was used to determine significance. When the P-values $\leq 0.05$, the differences were considered to be statistically significant.

\section{Results}

Characterization of the cell lines used for fusion. The $\mathrm{pDC}$ line PMDC05 displayed a characteristic phenotype, with easily detectable levels of HLA-ABC, HLA-DR, CD80 and CD86 but low levels of CD83 and very low levels of MUC1 (CD227) (Fig. 1A). Stimulation of this pDC line with LPS (LPS-pDC) resulted in the upregulated expression of HLA-ABC, HLA-DR,
CD80, CD86, CD83 and MUC1 (CD227) compared with unstimulated pDCs (Fig. 1A and B). Moreover, the LPS-pDCs exhibited increased levels of IL-12p70 and IL-10 compared with unstimulated pDCs (Fig. 1C). These results suggest that LPS activates pDCs. The pancreatic cancer cell lines used in this study, PANC-1 and MIA PaCa-2, expressed high levels of HLA-ABC and MUC1 but did not express HLA-DR, CD80, CD86 or CD83 (Fig. 2). Moreover, the PANC-1 cells expressed HLA-A2 but not HLA-A24, and conversely, the MIA PaCa-2 cells expressed HLA-A24 but not HLA-A2 (Fig. 2).

Characterization of the pDC/tumor fusion cells. To assess the capacity of the pDC/tumor fusion cells to induce antigenspecific CTL responses in vitro, we developed four types of fusion cell preparations by alternating fusion partners and treating with LPS. PANC-1 and MIA PaCa-2 cells were each successfully fused with pDCs with or without LPS stimulation (Fig. 3). The fusion efficiency was determined using the percentage of MUC1 and HLA-DR or CD86 double-stained cells (Fig. 3). Analysis of the fusion cells created from the pancreatic cancer cells and the pDCs demonstrated that about $50 \%$ of the population expressed both MUC1 and HLA-DR or CD86 (Fig. 3C and D). Interestingly, the fusions generated in the presence of LPS exhibited higher double-positive cells that expressed MUC1 and HLA-DR or CD86 than those generated with the unstimulated pDCs (Fig. 3C and D).

Next, to attain a detailed phenotypic characterization of the DC/tumor fusion cells, the mean fluorescence intensity (MFI) of HLA-DR and CD86 expression was determined by FACS analysis, where the fused cells were identified as MUC1 + HLA-DR ${ }^{+}$or MUC1 + $\mathrm{CD}^{2} 6^{+}$. Although the pDC/PANC-1 and pDC/MIA PaCa-2 cells displayed high MFI values for HLA-DR and CD86, the LPS-DC/PANC-1 and LPS-DC/MIA PaCa-2 cells exhibited higher MFI values on a per-fusion-cell basis (Fig. 4A). Therefore, fusions generated in the presence of LPS may have a more active phenotype compared to those generated with unstimulated pDCs.

Furthermore, we assessed the production of IL-12p70 and IL-10 in the supernatants from the fusion cell preparations. About 2-fold higher levels of IL-12p70 production were observed for the LPS-pDC/PANC-1 and LPS-pDC/MIA PaCa- 2 cells compared with the pDC/PANC- 1 and pDC/MIA PaCa-2 cells (Fig. 4B). Moreover, IL-10 production was also increased in the LPS-pDC/PANC-1 and LPS-pDC/MIA PaCa- 2 cells but to a lesser extent than that observed for IL-12p70 (Fig. 4B). Collectively, these results suggest that the upregulated production of IL-12p70 and the active phenotype of the fusion cells generated in the presence of LPS increase their immunogenicity.

Stimulation of $T$ cells by the pDC/tumor fusions. Although all four types of fusions affected $\mathrm{T}$ cell proliferation, the LPS-pDC/tumor fusion cells showed the most significant stimulation of $\mathrm{T}$ cell proliferation (Fig. 5A). In addition, an unfused mixture of tumor cells and pDCs or LPS-DCs had no effect on $\mathrm{T}$ cell proliferation (data not shown). Moreover, both the $\mathrm{pDC} /$ tumor and LPS-pDC/tumor fusions stimulated IFN- $\gamma$-producing $\mathrm{CD}^{+}$and $\mathrm{CD}^{+} \mathrm{T}$ cells (Fig. 5B). However, the LPS-pDC/tumor fusion cells more strongly induced the proliferation of both $\mathrm{CD} 4^{+}$and $\mathrm{CD} 8^{+} \mathrm{T}$ cells that were 
A
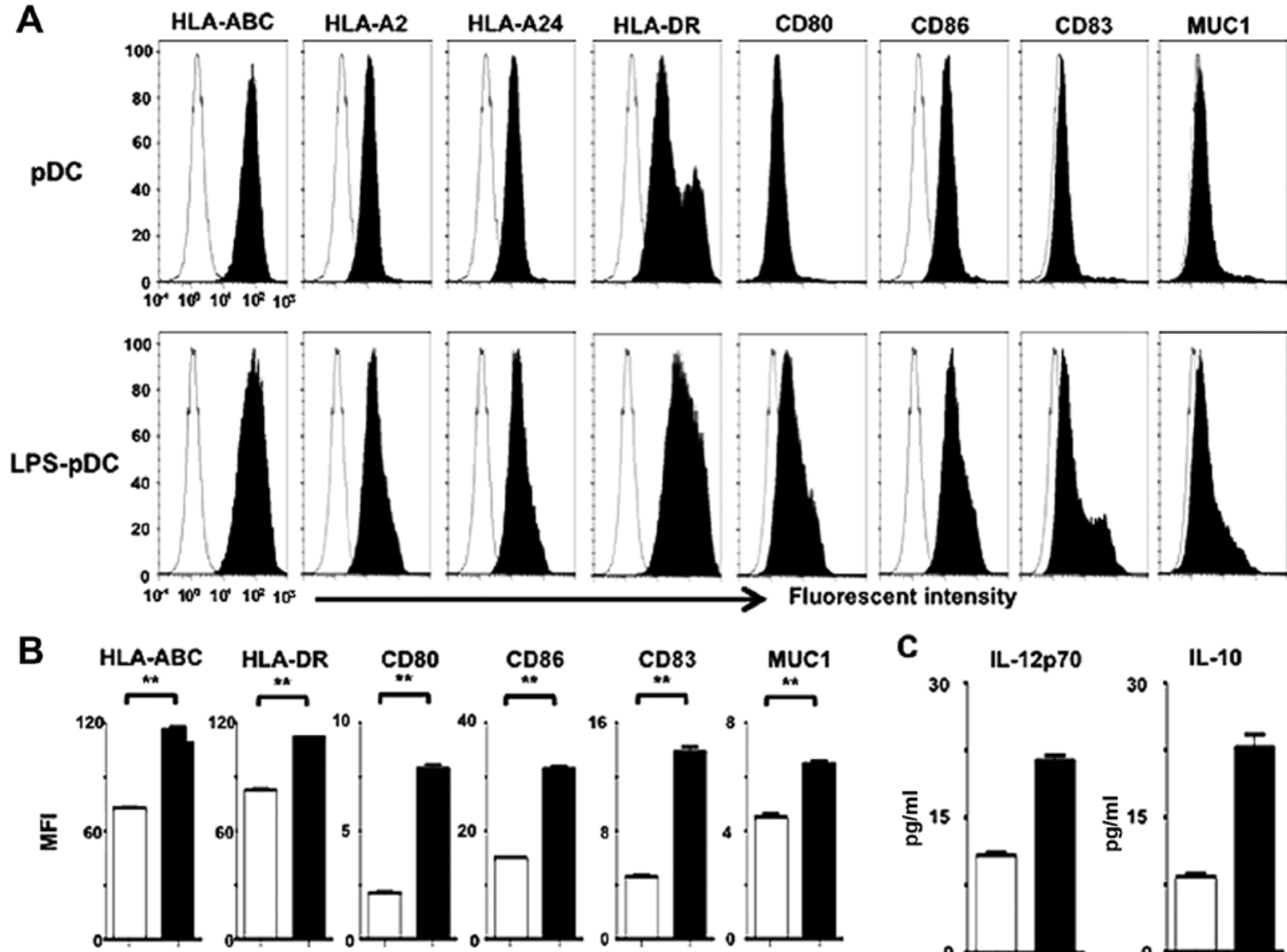

DDDC DLPS-PDC

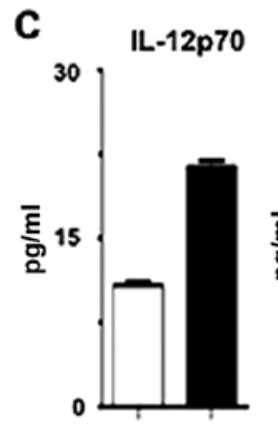

IL-10

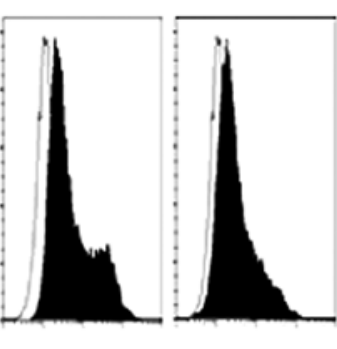

DPDC \LPS.PDC

Figure 1. Phenotypic and functional characterization of DCs. (A) Plasmacytoid dendritic cells (pDCs) and lipopolysaccharide (LPS)-stimulated pDCs (LPS-pDCs) were analyzed by flow cytometry for the expression of the indicated antigens. The unfilled histogram profile indicates the isotype control, and the solid histogram indicates the specific antibody. (B) The mean fluorescence intensity (MFI) for the expression of the indicated molecules in the pDCs and LPS-pDCs was determined ( $\mathrm{n}=3)$. (C) Production of IL-12p70 and IL-10 by the pDCs and LPS-pDCs $(\mathrm{n}=3)$ was analyzed by ELISA. The results are expressed as the mean $\pm \mathrm{SD} .{ }^{* * * *} \mathrm{P}<0.001 ;{ }^{* *} \mathrm{P}<0.01 ;{ }^{*} \mathrm{P}<0.05$.

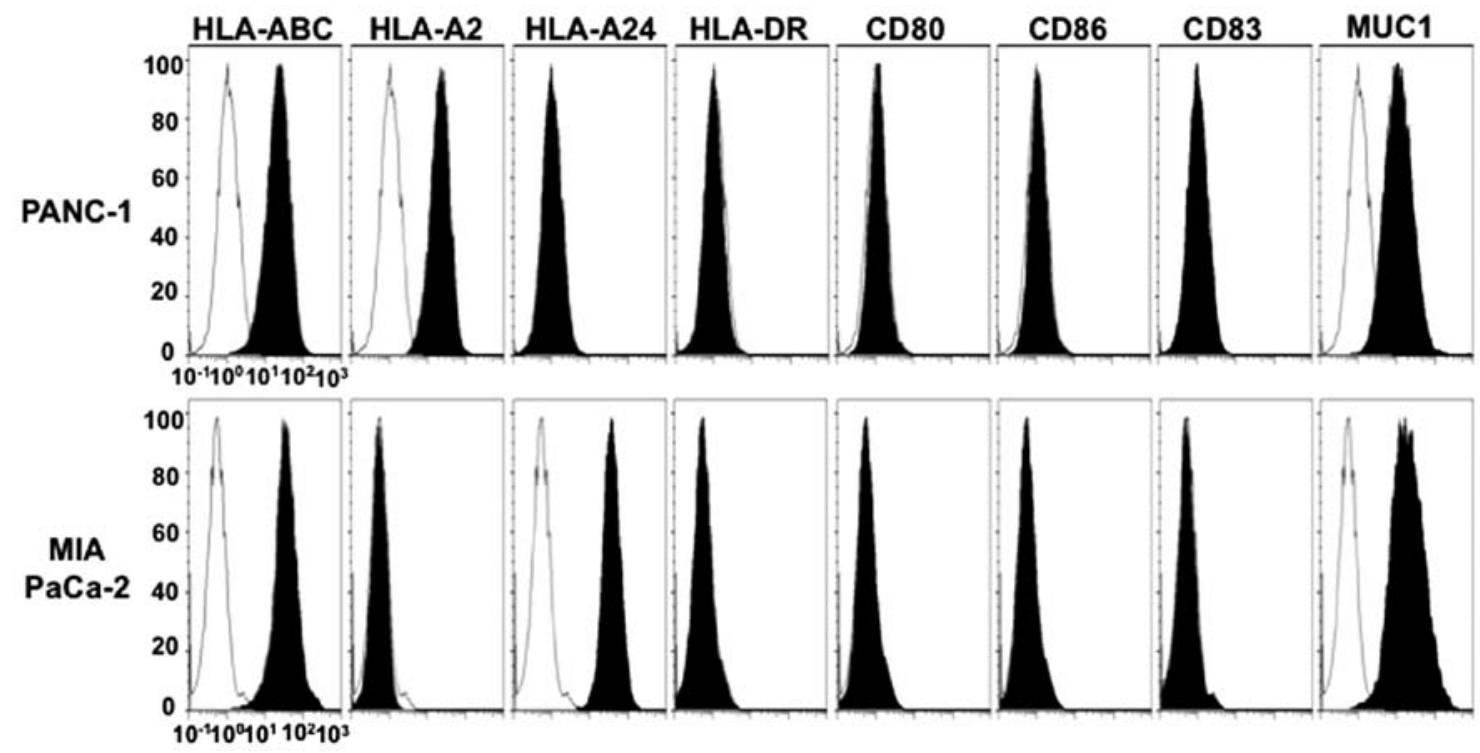

Figure 2. Phenotypic characterization of pancreatic tumor cells. PANC-1 and MIA PaCa-2 cells were analyzed by flow cytometry for expression of the indicated antigens. The unfilled histogram profile indicates the isotype control, and the solid histogram indicates the specific antibody. 

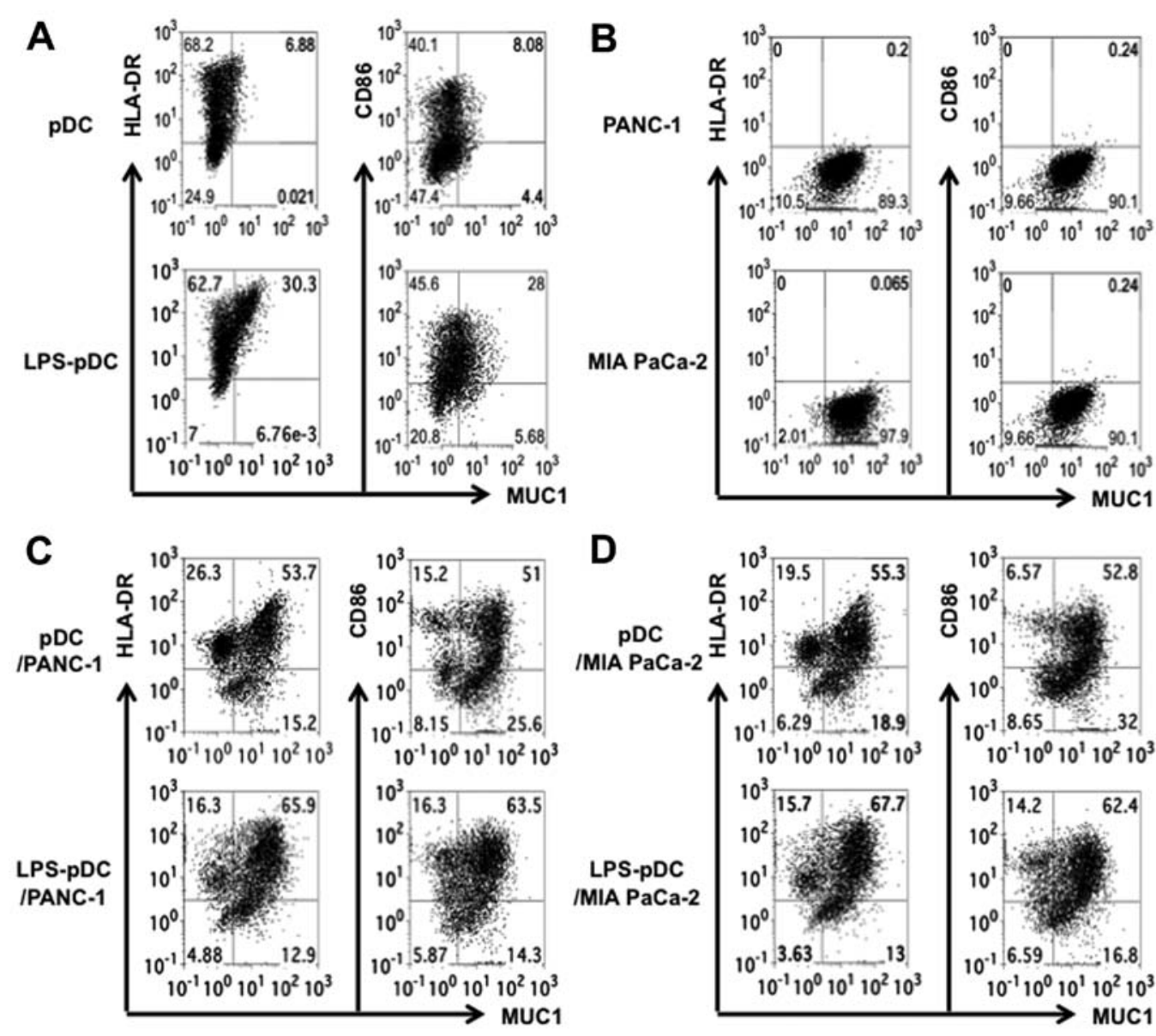

Figure 3. Phenotypic characterization of pDC/tumor fusion cells. (A) Plasmacytoid dendritic cells (pDCs) and lipopolysaccharide (LPS)-stimulated pDCs (LPS-pDCs), (B) PANC-1 and MIA PaCa-2 cells, (C) pDC/PANC-1 fusion cells and LPS-stimulated pDC/PANC-1 fusion cells and (D) pDC/MIA PaCa-2 fusion cells and LPS-stimulated pDC/MIA PaCa-2 fusion cells were stained with a FITC-conjugated mAb against MUC1 and PE-conjugated mAbs against HLA-DR and CD86 and then analyzed by two-color flow cytometry. The number of events in each region is shown.

capable of producing high levels of IFN- $\gamma$ compared to the pDC/tumor fusion cells (Fig. 5B). In contrast, very low levels or no IFN- $\gamma$-producing cells were detected in the $\mathrm{CD} 4^{+}$and $\mathrm{CD}^{+} \mathrm{T}$ cell populations stimulated by an unfused mixture of DCs and tumor cells (data not shown). These results suggest that $\mathrm{pDC} /$ tumor fusion cells stimulated with LPS have a more potent capacity to induce CTL responses compared to unstimulated $\mathrm{pDC} /$ tumor fusion cells.

MUC1-specific CTL responses induced by the pDC/tumor fusions. The CTLs induced by all four types of fusions lysed the tumor target cells used for fusion (Fig. 6A and B) but not K562 cells (data not shown). Moreover, the lytic activity induced by the LPS-stimulated pDC/tumor fusions was significantly higher than that induced by the unstimulated $\mathrm{pDC} /$ tumor fusions (Fig. 6A and B), suggesting that LPS increases the immunogenicity of the $\mathrm{pDC} /$ tumor fusion cells to induce efficient CTL responses. In addition, preincubation of the target cells with an anti-HL-ABC mAb inhibited their lysis, indicating restriction by MHC class I molecules (Fig. 6B). Interestingly, an increased percentage of HLA-A2-restricted, MUC1-specific $\mathrm{CD} 8^{+} \mathrm{T}$ cells in the whole $\mathrm{CD} 8^{+} \mathrm{T}$ cell population was observed for the LPS-pDC/tumor fusions (HLA-A2 ${ }^{+}$) compared with the $\mathrm{pDC} /$ tumor fusions (HLA-A2 $2^{+}$(Fig. 6C). In addition,
CTLs specific for MUC1 were not detected in a population of $\mathrm{T}$ cells stimulated by an unfused mixture of tumor cells and pDCs or LPS-pDCs (Fig. 6D). Together, these findings indicate that HLA-A2-restrictive, MUC1-specific CTLs are efficiently induced by LPS-pDC/tumor fusions in vitro.

\section{Discussion}

The data presented herein show that DC/tumor fusion cells generated with a pDC line (HLA-A2 ${ }^{+}$) and a pancreatic cancer cell line expressing MUC1 antigens induce HLA-A2-restricted, MUC1-specific CTLs in vitro. Moreover, LPS-stimulated pDC/tumor fusion cells efficiently induce augmented CTL responses.

We attempted to prepare immunogenic DC/tumor fusion cells using a DC line and a pancreatic cancer cell line. We used the plasmacytoid DC line PMDC05, a leukemic blast line that was isolated from a patient with acute leukemia $(11,12)$ and has been reported to have the capacity to induce effective antigen-specific CTLs $(13,14)$. This pDC line has been pulsed with peptide to induce CTL responses; however, little is known about its utility in cancer vaccines if used to generate $\mathrm{pDC} /$ tumor fusion cells. Cell lines that are well characterized can be massively propagated in vitro adhering to GMP. Thus, 

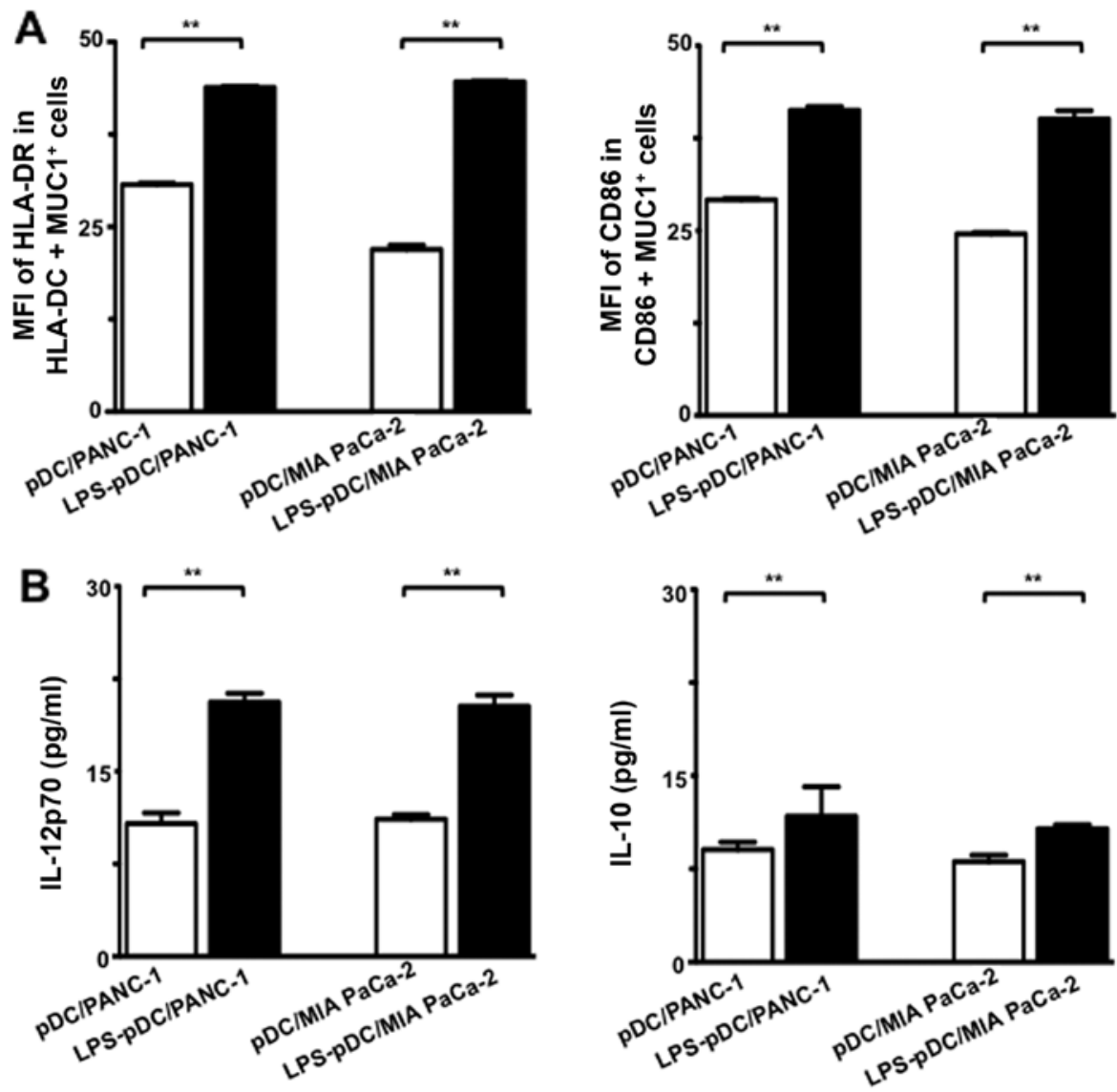

Figure 4. Functional characterization of pDC/tumor fusion cells. (A) Four types of fusions (plasmacytoid dendritic cell (pDC)/PANC-1, lipopolysaccharide (LPS)-stimulated pDC/PANC-1 (LPS-pDC/PANC-1), pDC/MIA PaCa-2, LPS-stimulated pDC/MIA PaCa-2 (LPS-pDC/MIA PaCa-2) (n=3) were stained with a FITC-conjugated mAb against MUC1 and PE-conjugated mAbs against HLA-DR and CD86 and then analyzed by FACS, where the fused cells were identified as MUC1 $+\mathrm{HLA}-\mathrm{DR}^{+}$or MUC1 $+\mathrm{CD} 86^{+}$. The mean fluorescence intensity (MFI) of the HLA-DR ${ }^{+}$and $\mathrm{CD}^{+} 6^{+}$cells was analyzed in the MUC1 + HLA-DR ${ }^{+}$and MUC1 + CD86 ${ }^{+}$populations, respectively. (B) The mean concentration of IL-12p70 and IL-10 produced by the four types of fusions $(\mathrm{n}=3)$ was analyzed by ELISA. The results are expressed as the mean $\pm \mathrm{SD} .{ }^{* * *} \mathrm{P}<0.001 ;{ }^{* * *} \mathrm{P}<0.01 ;{ }^{*} \mathrm{P}<0.05$.

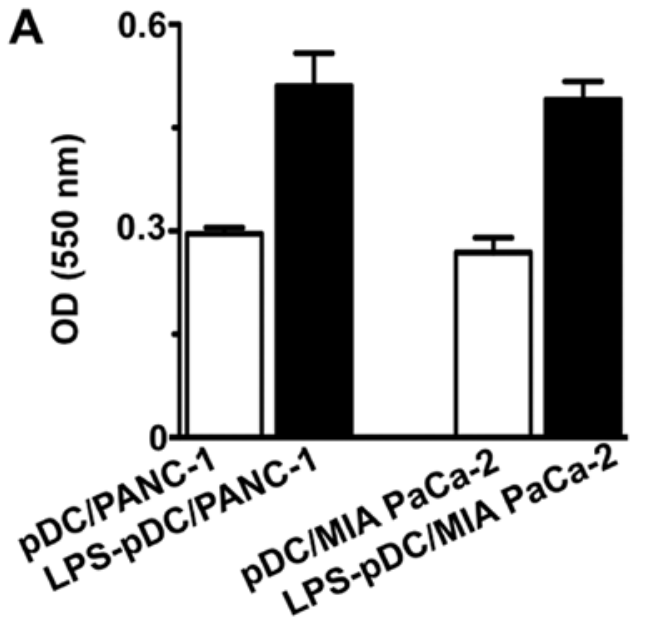

Figure 5. Activation of T cells by pDC/tumor fusions. (A) T cells were cocultured with each of the four types of fusions, and T cell proliferation was analyzed using the Cell Titer 96 Non-radioactive Cell Proliferation Assay kit according to the manufacturer's protocol. Similar results were obtained in three independent experiments. (B) T cells were stimulated with each of the four types of fusions in the absence of rhIL-2 for 3 days, maintained in the presence of low doses of rhIL-2 for 8 days and then assessed for IFN- $\gamma$ production by flow cytometry. Similar results were obtained in three independent experiments.

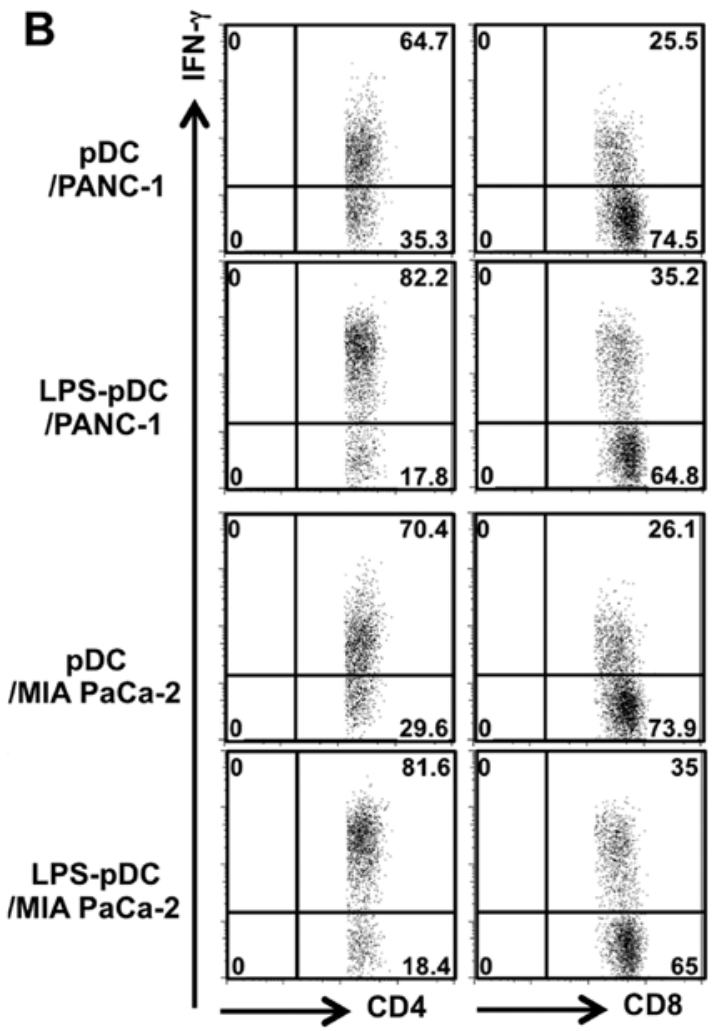



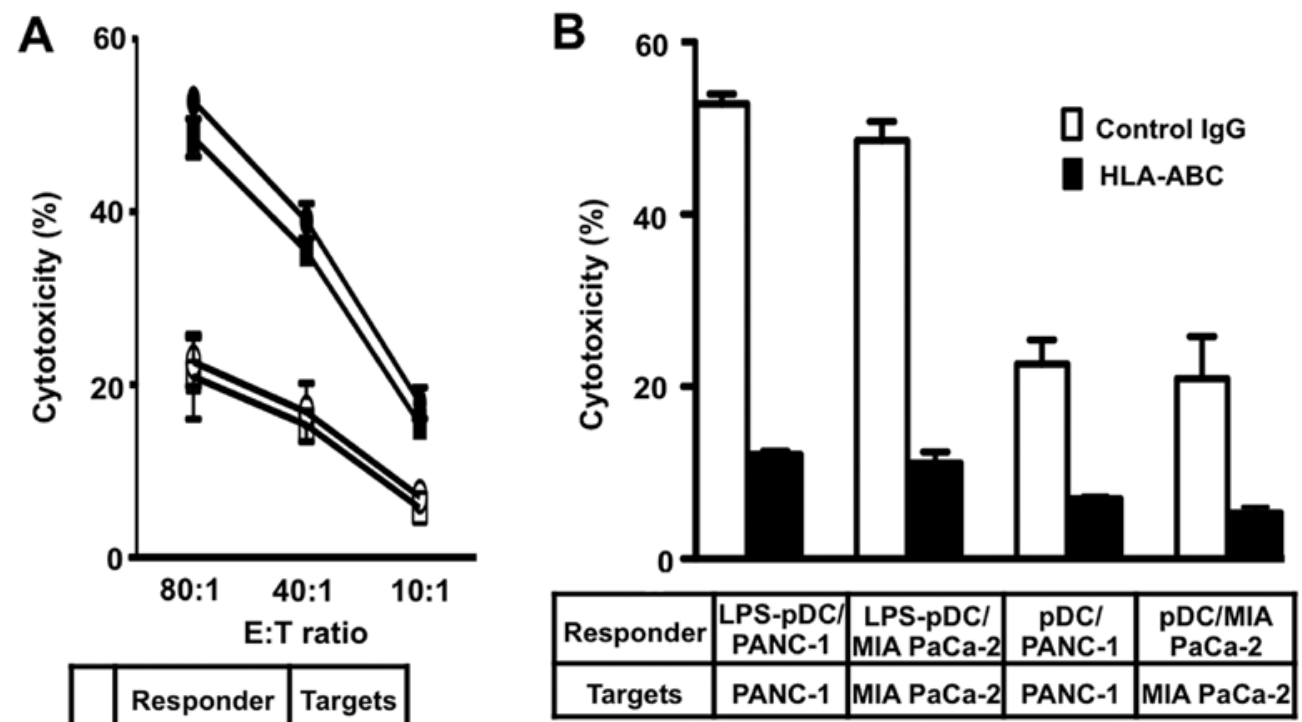

\begin{tabular}{|c|c|c|}
\hline & Responder & Targets \\
\hline 0 & pDC/PANC-1 & PANC-1 \\
\hline 0 & $\begin{array}{c}\text { LPS-pDC/ } \\
\text { PANC-1 }\end{array}$ & PANC-1 \\
\hline$\square$ & $\begin{array}{c}\text { pDC/MIA } \\
\text { PaCa-2 }\end{array}$ & $\begin{array}{c}\text { MIA } \\
\text { PaCa-2 }\end{array}$ \\
\hline & $\begin{array}{c}\text { LPS-pDC/ } \\
\text { MIA } \\
\text { MIA PaCa-2 }\end{array}$ & \begin{tabular}{c} 
PaCa-2 \\
\hline
\end{tabular} \\
\hline
\end{tabular}

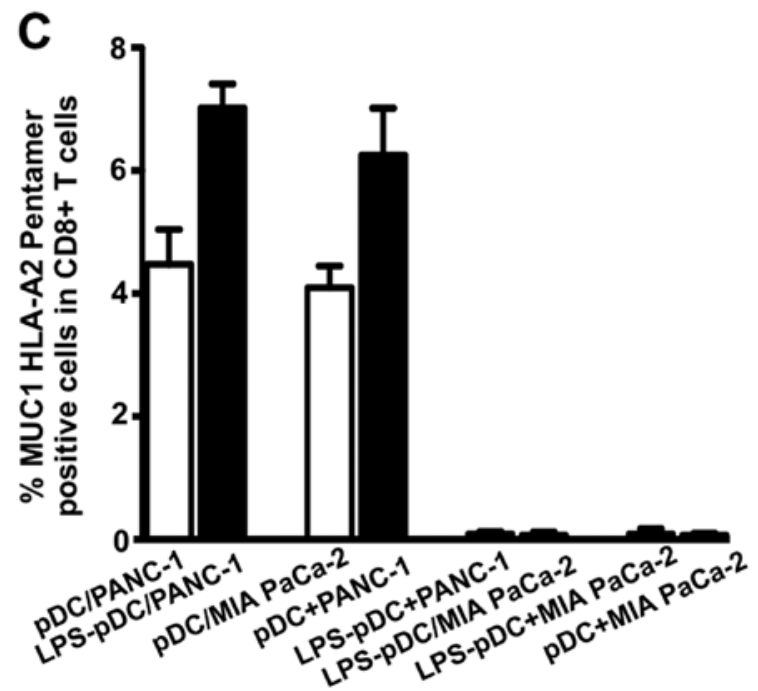

Figure 6. Induction of MUC1-specific CTL responses against tumor targets by $\mathrm{pDC} /$ tumor fusions. (A) T cells (n=3) were stimulated with each of the four types of fusions, which coexpressed both MUC1 and HLA-DR. The stimulated T cells were then incubated with PKH-26-labeled target tumor cells at the indicated effector cell:T cell (E:T) ratios (80:1,40:1, 10:1) for cytotoxicity assays. (B) Stimulated T cells $(\mathrm{n}=3)$ were cocultured with PKH-26-labeled target cells at a ratio of 80:1. The target cells were preincubated with control IgG or a mAb against HLA-ABC (W6/32; 1:100 dilution). The percentage of cytotoxicity (mean \pm SD) was determined by a flow cytometry-based CTL assay. (C) The percentage of $\mathrm{CD} 8^{+} \mathrm{T}$ cells that reacted with the MUC1 pentamer among the whole $\mathrm{CD} 8^{+} \mathrm{T}$ cell population (n=3) is shown as a percentage of double-positive cells $\left(\mathrm{MUC1}\right.$ pentamer $\left.+\mathrm{CD} 8^{+}\right)$in the total $\mathrm{CD} 8^{+} \mathrm{T}$ cell population. The results are expressed as the mean $\pm \mathrm{SD}$.

unlimited amounts of DC/tumor fusion cells can be readily available to induce antigen-specific CTLs for adoptive immunotherapy. Therefore, one important aspect of our work is its potential clinical relevance.

The binding of the pathogen-associated microbial pattern molecule LPS to Toll-like receptor (TLR) 4 on human MoDCs signals danger, which induces a potent immune stimulatory phenotype that is characterized by the release of IL-12p70 $(18,19)$. Our finding that a pDC line activated with LPS is more active compared to unstimulated pDCs suggests that this TLR4 agonist plays a role in the activation of pDC functions (11). Moreover, LPS stimulation resulted in increased production of both IL-12p70 and IL-10 by the pDCs. The surface phenotype and cytokine production response pattern of the pDCs in this study was similar to that of human MoDCs (20), which implies that this pDC line possesses characteristics of MoDCs (11).
Moreover, stimulation of the pDC line with LPS resulted in considerably increased expression of MUC1 (CD227) on the cell surface. MUC1 (CD227) is considered to be an epithelial mucin that is expressed extensively in pancreatic and other cancer types; thus, MUC1 is a target for immunotherapy in a variety of cancers (21). This molecule is also expressed by a wide variety of hemopoietic cells, from early differentiating bone marrow mononuclear cells to mature cell types (22). It is also known that MUC1 (CD227) is expressed by activated DCs and T cells (23). Therefore, LPS-stimulated pDCs, which express increased levels of MUC1 (CD227), HLA-ABC, -DR, CD80, CD86, CD83 and IL-12p70, may be suitable for cancer vaccines. Therefore, we speculated that fusion cells generated with a pDC line and a tumor cell line in the presence of a TLR4 agonist would be immunogenic and induce more effective MUC1-specific CTLs than their unstimulated counterparts. 
We successfully fused a pDC line with two different tumor cell lines with or without LPS stimulation. The pDC/tumor fusion cells were identified as MUC1 + HLA-DR ${ }^{+}$or MUC1 + CD86 ${ }^{+}$. The characteristic phenotype of the LPS-stimulated pDCs was associated with an increased percentage of double-positive cells $\left(\mathrm{MUC1}+\mathrm{HLA}-\mathrm{DR}^{+}\right.$or $\left.\mathrm{MUC1}+\mathrm{CD} 86^{+}\right)$in the $\mathrm{pDC} /$ tumor fusion cell preparations (data not shown). Moreover, the cells that were double positive for MUC1 and HLA-DR or CD86 in the LPS-stimulated pDC/tumor fusion cell preparations had high MFI values for HLA-DR and CD86 on a per-fusion-cell basis, indicating that the fusions were more immunogenic compared to their unstimulated counterparts. Our previous report demonstrated that efficient CTL induction is closely correlated to fusion efficiency for fusion cells generated with MoDCs (24). LPS might provide the costimulation required during the fusion process and might be involved in polarizing the $\mathrm{T}$ cell responses to a Th1-dominant state. Therefore, the efficient activation of the pDC/tumor fusion cells by LPS led us to speculate that the MUC1-specific CTLs induced by these activated fusion cells would be more effective than conventional unactivated fusion cells.

We previously reported that the tumor antigens delivered to MoDCs by fusion cells were processed and presented in the context of MHC class I and II molecules of MoDC origin of fusion cells $(15,25)$. Therefore, the HLA typing of the MoDCs and allogeneic tumor cell lines does not need to match (26). HLA-A2-restricted, MUC1-specific CTLs were efficiently generated with the fusion cells generated from allogeneic pDC (HLA-A2 ${ }^{+}$) and MIA PaCa-2 (HLA-A'), suggesting that the MUC1 antigens from the MIA PaCa-2 cells were also processed and presented by HLA-A2 on the pDC part of $\mathrm{pDC} /$ tumor fusion cells. Although the LPS-pDC/tumor and $\mathrm{pDC} /$ tumor fusions stimulated IFN- $\gamma$-producing $\mathrm{CD}^{+}$and $\mathrm{CD}^{+} \mathrm{T}$ cells that lyse the tumor target cells used for fusion, the LPS-pDC/tumor fusions more strongly induced T cell activation, indicating that LPS stimulation is effective for $\mathrm{pDC} /$ tumor fusion cell vaccines. Moreover, the MUC1-specific CTLs were more effectively augmented by the LPS-pDC/tumor fusion cells compared to the $\mathrm{pDC} /$ tumor fusion cells. These results may be associated with the active function of LPS-DCs as PACs, as demonstrated by their mature phenotype, IL-12p70 production and increased MUC1 expression. In patients with melanoma or renal cell carcinoma, vaccines using fusions of allogeneic MoDCs and autologous tumor cells have been shown to induce efficient antitumor immune responses and clinical outcomes $(27,28)$. Moreover, allogeneic tumor cell lines have been used in fusion cell vaccines in both preclinical $(25,29,30)$ and clinical studies (31) and a MoDC/tumor fusion cell vaccine with fully allogeneic components has been demonstrated to induce clinical responses (31). Therefore, DC/tumor fusions generated with fully syngeneic, semi-allogeneic or fully allogeneic components are effective in inducing antigen-specific, long-lasting antitumor immunity (32).

In conclusion, our results indicate that fusion cell vaccines generated with a plasmacytoid DC line and tumor cell line can induce antigen-specific CTL responses in vitro. Our findings introduce the possibility of using defined allogeneic plasmacytoid DC and tumor lines to simplify CTL manufacturing for adoptive immunotherapy.

\section{Acknowledgements}

This study was supported by Grants-in-Aid for Scientific Research (C) from the Ministry of Education, Cultures, Sports, Science and Technology of Japan, the Foundation for Promotion of Cancer Research, the Mitsui Life Social Welfare Foundation, and a Grant-in-Aid from the Japan Medical Association. The funders had no role in the study design, data collection or analysis, decision to publish or manuscript preparation.

\section{References}

1. Steinman RM: The dendritic cell system and its role in immunogenicity. Annu Rev Immunol 9: 271-296, 1991.

2. Gong J, Chen D, Kashiwaba M and Kufe D: Induction of antitumor activity by immunization with fusions of dendritic and carcinoma cells. Nat Med 3: 558-561, 1997.

3. Gong J, Koido S and Calderwood SK: Cell fusion: from hybridoma to dendritic cell-based vaccine. Expert Rev Vaccines 7: 1055-1068, 2008.

4. Koido S, Homma S, Okamoto M, et al: Fusions between dendritic cells and whole tumor cells as anticancer vaccines. Oncoimmunology 2: e24437, 2013.

5. Yanagimoto H, Takai S, Satoi S, et al: Impaired function of circulating dendritic cells in patients with pancreatic cancer. Clin Immunol 114: 52-60, 2005.

6. Colonna M, Trinchieri G and Liu YJ: Plasmacytoid dendritic cells in immunity. Nat Immunol 5: 1219-1226, 2004.

7. Liu YJ: IPC: professional type 1 interferon-producing cells and plasmacytoid dendritic cell precursors. Annu Rev Immunol 23: 275-306, 2005.

8. Kim R,Emi M, Tanabe K and Arihiro K: Potential functional role of plasmacytoid dendritic cells in cancer immunity. Immunology 121: 149-157, 2007.

9. Segura E, Kapp E, Gupta N, et al: Differential expression of pathogen-recognition molecules between dendritic cell subsets revealed by plasma membrane proteomic analysis. Mol Immunol 47: 1765-1773, 2010.

10. Mouries J, Moron G, Schlecht G, Escriou N, Dadaglio G and Leclerc C: Plasmacytoid dendritic cells efficiently cross-prime naive T cells in vivo after TLR activation. Blood 112: 3713-3722, 2008.

11. Narita M, Watanabe N, Yamahira A, et al: A leukemic plasmacytoid dendritic cell line, PMDC05, with the ability to secrete IFN-alpha by stimulation via Toll-like receptors and present antigens to naive T cells. Leuk Res 33: 1224-1232, 2009.

12. Watanabe N, Narita M, Yamahira A, et al: Transformation of dendritic cells from plasmacytoid to myeloid in a leukemic plasmacytoid dendritic cell line (PMDC05). Leuk Res 34: 1517-1524, 2010.

13. Yamahira A, Narita M, Nakamura T, et al: Generation of antigenspecific cytotoxic $\mathrm{T}$ lymphocytes using a leukemic plasmacytoid dendritic cell line as antigen presenting cells. Leuk Res 35: 793-799, 2011.

14. Yamahira A, Narita M, Ishii K, et al: Enhancement of antigen presenting ability in the leukemic plasmacytoid dendritic cell line (PMDC05) by lentiviral vector-mediated transduction of CD80 gene. Leuk Res 36: 1541-1546, 2012.

15. Koido S, Hara E, Homma S, et al: Dendritic/pancreatic carcinoma fusions for clinical use: comparative functional analysis of healthyversus patient-derived fusions. Clin Immunol 135: 384-400 2010.

16. Koido $\mathrm{S}$ and Gong J: Characterization of structure and direct antigen presentation by dendritic/tumor-fused cells as cancer vaccines. Anticancer Res 33: 347-354, 2013.

17. Liu L, Chahroudi A, Silvestri G, et al: Visualization and quantification of $\mathrm{T}$ cell-mediated cytotoxicity using cell-permeable fluorogenic caspase substrates. Nat Med 8: 185-189, 2002.

18. Lapteva N, Seethammagari MR, Hanks BA, et al: Enhanced activation of human dendritic cells by inducible CD40 and Toll-like receptor-4 ligation. Cancer Res 67: 10528-10537, 2007.

19. Luger R, Valookaran S, Knapp N, Vizzardelli C, Dohnal AM and Felzmann T: Toll-like receptor 4 engagement drives differentiation of human and murine dendritic cells from a pro- into an anti-inflammatory mode. PLoS One 8: e54879, 2013. 
20. Koido S, Homma S, Okamoto M, et al: Combined TLR2/4 activated dendritic/tumor cell fusions induce augmented cytotoxic T lymphocytes. PLoS One 8: e59280, 2013.

21. Kimura T and Finn OJ: MUC1 immunotherapy is here to stay. Expert Opin Biol Ther 13: 35-49, 2013.

22. Brugger W, Buhring HJ, Grunebach F, et al: Expression of MUC-1 epitopes on normal bone marrow: implications for the detection of micrometastatic tumor cells. J Clin Oncol 17: 1535-1544, 1999.

23. Wykes M, MacDonald KP, Tran M, et al: MUC1 epithelial mucin (CD227) is expressed by activated dendritic cells. J Leukoc Biol 72: 692-701, 2002.

24. Koido S, Hara E, Homma S, et al: Streptococcal preparation OK-432 promotes fusion efficiency and enhances induction of antigen-specific CTL by fusions of dendritic cells and colorectal cancer cells. J Immunol 178: 613-622, 2007.

25. Koido S, Hara E, Homma S, et al: Dendritic cells fused with allogeneic colorectal cancer cell line present multiple colorectal cancer-specific antigens and induce antitumor immunity against autologous tumor cells. Clin Cancer Res 11: 7891-7900, 2005.

26. Koido S, Hara E, Homma S, Ohkusa T, Gong J and Tajiri H: Cancer immunotherapy by fusions of dendritic cells and tumor cells. Immunotherapy 1: 49-62, 2009.
27. Haenssle HA, Krause SW, Emmert S, et al: Hybrid cell vaccination in metastatic melanoma: clinical and immunologic results of a phase I/II study. J Immunother 27: 147-155, 2004.

28. Trefzer U, Herberth G, Wohlan K, et al: Tumour-dendritic hybrid cell vaccination for the treatment of patients with malignant melanoma: immunological effects and clinical results. Vaccine 23: 2367-2373, 2005.

29. Lundqvist A, Palmborg A, Bidla G, Whelan M, Pandha H and Pisa P: Allogeneic tumor-dendritic cell fusion vaccines for generation of broad prostate cancer T-cell responses. Med Oncol 21: 155-165, 2004.

30. Matsumoto S, Saito H, Tsujitani S and Ikeguchi M: Allogeneic gastric cancer cell-dendritic cell hybrids induce tumor antigen (carcinoembryonic antigen) specific CD8(+) T cells. Cancer Immunol Immunother 55: 131-139, 2006.

31. Marten A, Renoth S, Heinicke T, et al: Allogeneic dendritic cells fused with tumor cells: preclinical results and outcome of a clinical phase I/II trial in patients with metastatic renal cell carcinoma. Hum Gene Ther 14: 483-494, 2003.

32. Siders WM, Garron C, Shields J and Kaplan JM: Induction of antitumor immunity by semi-allogeneic and fully allogeneic electrofusion products of tumor cells and dendritic cells. Clin Transl Sci 2: 75-79, 2009. 